\title{
كفاءات معلمي اللغة العربية في إندونيسيا في القرن الحادي والعشرين
}

\author{
Cahya Edi Setyawan \\ STAI Masjid Syuhada Yogyakarta \\ cahya.edi24@gmail.com \\ Akhsan \\ Universitas Ibrahimy Sukorejo Situbondo \\ ahsanthayyib@gmail.com
}

\begin{abstract}
In the 21 st century is a disruptive era, millennial era, digital age. Information technology and science develop. This development was followed by the development of the human generation, namely generazi Z. This generation is technology literate, multitalent, and creative. In the world of education of course this makes a very extraordinary impact. Arabic language teachers as one of the components of education must follow the flow of globalization. Arabic teachers face many challenges in this era. Therefore there is a standard for Arabic teacher competencies. In the standardization, of course there are efforts made by the government, agencies, institutions and individual teachers in developing and training their competencies. Therefore this paper is a literary paper that discusses how the challenges faced by Arabic language teachers in the 21st century, then the competency standards of 21 st century Arabic language teacher as well as the efforts made to develop them.
\end{abstract}

Keywords: 21 st century, compotence of arabic language teachers 
وتعدد المهام .جيل الألفية بطلاقة للغاية في

التكنولوجيا, والدهاء على شبكة الإنترنت, جيل الودية. هم الجيل الرقمي الذين يتقنون ويفتنون بتقنية المعلومات وتطبيقات الكمبيوتر المختلفة. إ فم يتفاعلون بشدة عبر وسائل التواصل الاجتماعي مع جميع الفئات. إفم مكثفون للغاية في التواصل والتفاعل مع جميع المجموعات, وخاصة مع أقراهم من خلال مواقع الشبكات المختلفة, مثل:FaceBook أو مئ مُأو عبر الرسائل القصيرة. إغم يميلون إلى التسامح مع الاختلافات الثقافية وهم مهتمون جأًا بالبيئة،وهم معتادون على الأنشطة المختلفة

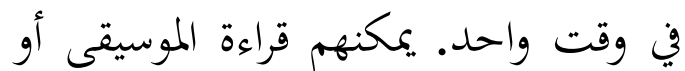

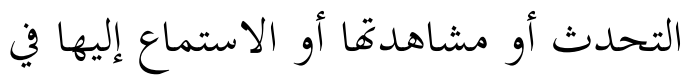
نفس الوقت. إفم يريدون كل شيء يجب القيام به وتشغيل شريع. إذم لا يريدون أشياء طويلة الأمد وملتوية. هذا بالتأكيد يصبح مشكلة للمعلمين. يحب أن يكون المعلمون قادرين على ضبط مهاراتم وبتهيز أنفسهم بالكفاءات المختلفة, خاصة تلك الموجودة في بجالات التكنولوجيا واللغة.

Bahasa Arab (PINBA) V di Bandung pada tanggal 23-25 Agustus 2007, hlm. 5

Imtiyaz: Jurnal Pendidikan dan Bahasa Arab https://ejournal.iainbengkulu.ac.id/index.php/imtiyaz
إن القرن الحادي والعشرون تختلف مع

القرون السابقة. إن تسارع (sophisticated) تطور العلوم في مجال تكنولوجيا المعلومات والاتصالات (ICT) يجعل مشاكله الخاصة في جوهر التعليم, على الرغم من أنه يسهل على الطلاب الوصول إلى المواد والعلوم.' هذه التغييرات محسوسة في عالم التعليم. يواجه المعلمون اليوم تحديات أكبر مما كانت عليه في العصور السابقة. يواجه المعلم الطلاب الأكثر تنوعًا, والموضوعات الأكثر تعقيدًا وصعوبة, ومستوى عملية التعليم ويطالب أيضًا بتحقيق قدرات التفكير العليا لدى الطلاب, وذلك لأن المدرسين بحاجة إلى أن يكونوا قادرين على المنافسة لم يعد

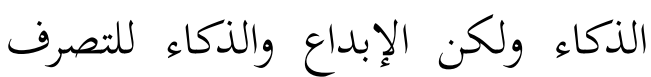
.(hard-skill and soft skill) وجود جيل الألفية يخلق مشاكله الخاصة للمعلمين. يجبأن يكون المعلم قادرًا على ضبط احتياجات طلاب الألفية في

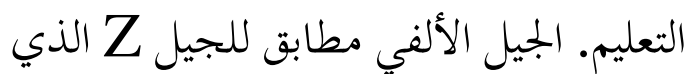
يعرف القراءة والكتابة والإبداع والتعبير

1 Emzir, ,Kebijakan Pemerintah Tentang Pembelajaran Bahasa Arab di Madrasah dan Sekolah Umum', Makalah disampaikan dalam Pertemuan Ilmiah Nasional 
كفاءات المعلمين من جميع جوانب الكفاءات التربوية والشخصية واللغوية. هناك سبعة تحديات للمدرسين في القرن الحادي والعشرين، وهي: ( ) التدريس Teaching in في مجتمع متعدد الثقافات (multicultural society المجتمعات التي لديها ثقافات متنوعة مع الكفاءات متعددة اللغات, Y ) تدريس لبناء construction of e Teaching for th المعنى r التدريس من أجل التعلم (meaning,

(؟ Teaching for active learning النشط Teaching and التدريب والتكنولوجيا (technology, 0 جديدة حول القدرات Teaching with ,Teaching and new view about abilities ד) التدريس والاختيار Teaching and choic, ح accountability التدريب والمساءلة لمواجهة التحديات المذكورة أعلاه، يتعين على معلمي اللغة العربية أن يكونوا

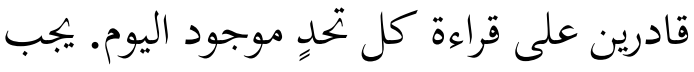

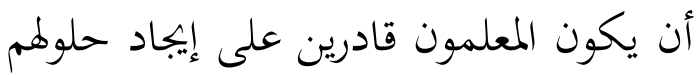

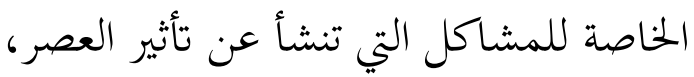

Radikalisme dan Berita Hoaks di Media Sosial. In: International Seminar on Islamic Studies. 2019 ${ }^{3}$ Suyanto, Tantangan profesional guru di era global, Pidato Dies Natalis ke 43, UNY, 2007. hlm. 2

Imtiyaz: Jurnal Pendidikan dan Bahasa Arab https://ejournal.iainbengkulu.ac.id/index.php/imtiyaz
يواجه التحدي أيضًا إلى مدرسي اللغة

العربية. و وكذلك بنسبة تحديات الثورة

الصناعية (.., (؟) تتطلب أن يتطور الناس

لمتابعة تقدم العلم والتكننولوجيا.` وتطور

التكنولوجي يتطلب المعلمين ماهرين في

تكنولوجيا. يجب أن يكون المعلمون قادرين

على تقديم المواد التعليمية باستخدام التكنولوجيا. على سبيل المثال: يقدم المعلم

التصميم المادي بمساعدة شاشة LCD و

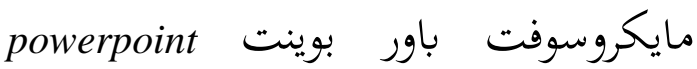
جذابة بحيث يكون الطلاب متحمسين للاستماع إلى الدرس والاهتمام به. يوفر المعلم المعلومات للطلاب حتى يتمكنوا من الوصول إلى اللغات من خلال تطبيق القاموس على بلى سلى الإنترنت أو تطبيق اللغة العربية على نظام اندرايد. Android يجب أن يبتكر المعلمون أكثر من ذلك للقيام بالتعليم عبر الإنترنت حتى لا يتعلم الطلاب بالملل بشكل مستمر

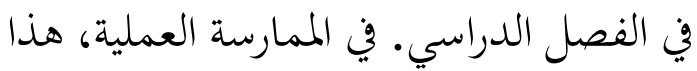

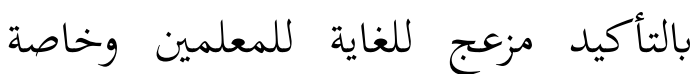
للمعلمين من كبار السن. يجب ترقية

2 Ixsir Eliya, Achmad Ja'far Sodik, Penguatan Keilmuan Mahasiswa Melalui Gerakan Sadar Literasi dalam Upaya Menangkal 
الكفاءة هي القدرة الكلية للشخص، أي أن القدرة على التعليم والمعرفة لا شك فيها. إذا تم عرضه من جانب الأداء ونتائج العمل، فلا

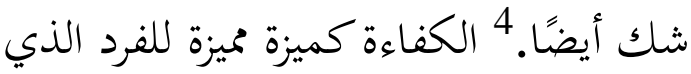
يرتبط بالأداء الفعال أو المتفوق في الوظيفة أو لميره كميزه الموقف. قدرة الشخص في مجال معين هي بلا شك أيقونة وموثوق بها.

توجّه معلمي القرن الحادي والعشرين وفقًا للعالم الدولي نحو مهمة المعلمين الذين يدخلون القرن الحادي والعشرين هو أن يكونوا قادرين على تنظيم عملية تعلّم ترتكز وتنفذ أركان التعلم الأربعة التي أوصت بها لجنة لمعند اليونسكو الدولية للتربية، وهذا يعتمد على أن التعليم هو اتصال منظم ومستدام. لتعزيز أنشطة التعلم لدى الطلاب (التعليم كاتصال منظم ومستدام مصمم لتحقيق التعلم). توصي اليونسكو UNESCO بأربع ركائز في مجال التعليم وهي: ا ( التعلم للمعرفة وتئ تعلم المعرفة هي عملية تعلم معرفة وفهم وتعيش طرق اكتساب المعرفة والتعليم التي تزود الطلاب بأحكام المعرفة. تتيح عملية التعلم هذه للطلاب القدرة على

${ }^{5}$ H. Hamzah, Profesi Kependidikan, Jakarta: Bumi Aksara, 2014, hlm. 9
لأنه ليس كل تقدم العصر له تأثير جيد، يجب أيضًا مراعاة الآثار السلبية. تشير صياغة المشكلة إلى ثلاثة أشياء، وهي: () التحديات التي تواجه معلمي اللغة العربية بإندونيسيا في القرن الحادي والعشرين في إندونيسيا

Y) معايير كفايات معلمي اللغة العربية بإندونيسيا في القرن الحادي والعشرين في إندونيسيا r) الجهود التي يمكن بذها في تطوير كفاءات معلمي اللغة العربية في إندونيسيا بـانيا

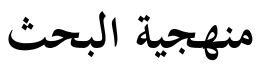

هذه المقالة هي مقالة مفاهيمية

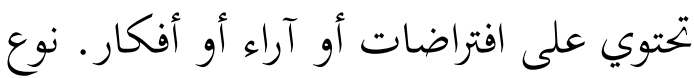
البحث هو البحث المكتبي. بالنسبة لطريقة جمع البيانات من خلال البحث عن مقالات أو كتب أو بجلات متعلقة بموضوع أو عنوان

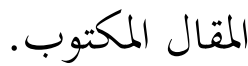

\section{توجيه المعلم في القرن الحادي والعشرين}

الكفاءة هي مسألة تصف مؤهلات

الشخص وقدراته، سواء النوعية والكمية. من

التعريف الذي قدمه عثمان، يتضح أن

4 Kunandar, Guru Profesional, Jakarta: Rajawali Press, 2007, hlm. 13 
الأصليين وأن يكونوا قادرين على قول ما سمعوه وأن يكتبوه، يجب أن يكون الطلاب قادرين على التحدث في كل دور، أي يجب أن يكون الطلاب قادرين على إلقاء الخطب والوعظ وقراءة الأخبار وإخبار شيء باستخدام اللغة العربية. "ا. التعلم للعيش معاء باستحا

تعلم العيش معا، أي التعليم يجب

أن يوفر القدرة على العيش معا في بجتمع تعددي من أجل خلق حياة سلمية وتسامح بين البشر. مع الأحكام التي حصل عليها الطلاب في المدارس، يكون الطلاب قادرين على تطبيق اللغة العربية في الحياة في المجتمع عندما يكون خريجًا. الطلاب قادرون على تدريس اللغة العربية

$$
\text { ع. التعلم لتطوير النفس لبئتهم المحتاجة. }
$$

يجب التعلم أن يكون توفير القدرة

على تطوير أنفسهم. تتيح عملية التعلم إنشاء طلاب مستقلين، لديهم ثقة بالنفس، قادرين على معرفة أنفسهم، فهم الذات، تحقيق الذات أو التوجيه الذاتي، لديهم قدرات عاطفية وفكرية ثابتة، والوصول إلى مستوى ثابت ومستقل للشخصية. من المتوقع أن تكون ابحاهات
معرفة المعلومات و أو العثور على المعرفة وفهمها وتطبيقها والبحث عنها. عند تعلم اللغة العربية، يجب على المعلم أن يعلم الطلاب كيفية فهم النصوص والتعبيرات العبية ومن ثم يكونوا قادرين على إيجاد معاني ومعاني هذه الكلمات والتعبيرات ويكونوا قادرين على ممارسة النصوص والتعبيرات في المحادثات وأنشطة الكتابة

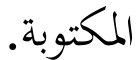
ץ. التعلم للقيام به (تعلم القيام به أو القيام

تعلم القيام به، وهي عملية تعلم القيام أو القيام بشيء ما. تعلم العمل والقيام بشيء ما يعني أن التعليم يجب أن أن

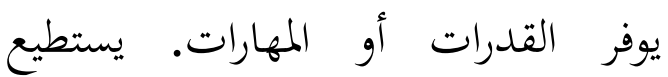
الطلاب في عملية التعلم استخدام مفاهيم أو مبادئ أو قوانين مختلفة لحل المشكلات الملموسة. في ممارسة تعلم اللغة العربية، يجب أن يكون المعلمون قادرين على تصميم الطلاب ليريدوا تطبيق اللغة بعبارات حقيقية، فهم ليسوا كافيين لفهم ومعرفة، لكنهم قادرون على ممارسة أو حل حل المشكلات في المشكلات اللغوية التي يواجهوها. على سبيل المثال: إفم قادرون على الاستماع إلى محادثات المتحلثين 
مدرسو اللغة العربية في إندونيسيا بتدريس القواعد العربية وتعزيزها أكثر من اللغة العربية التحادثية أو التطبيقية. لا يزال مدرسو اللغة العربية في إندونيسيا ضعيفين في إتقاهم للمفردات العربية وأنماط التعبير والترتيبات اللغوية. هذا لأن مدرسي اللغة

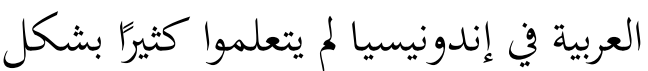

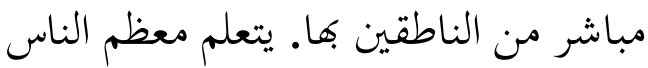
في إندونيسيا اللغة العربية مع الشعب الإندونيسي نفسه. لا يزال هناك العديد من المعلمين الذين يترددون في التحدث باللغة العربية عند التدريس وعند التحدث برثي يوميًا في المدرسة وذلك بسبب عدم وجود بيئة تتحدث اللغة العربية في المدرسة. نماذج التعلم باللغة العربية في المدارس لا تزال تركز على تعلم القرعة. أثناء الاستماع

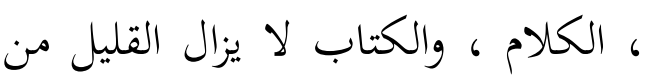

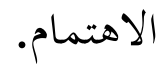

- تطوير التكنولوجيا ، وهي العصر

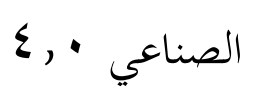

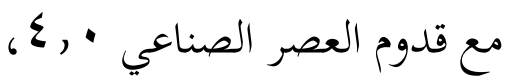
أثر ذلك على تطوير نماذج تعلم اللغة العربية عبر الإنترنت وعبر الإنترنت. كان هنان تحول من نموذج التعلم في الفصول الدراسية القائمة على الإنترنت والتكنولوجيا عبر
الإخراج قادرة على أن يصبحوا مدرسين للغة العربية، وأن يكونوا قادرين على ممارسة الأعمال التجارية في مجال اللغة العربية مثل تحارة الكتب العربية، وأن يكون الطلاب قادرين على فتح مؤسسات دورة اللغة العربية لكل من الأكاديميين والممارسين، مؤسيات دوره وأن يكون الطلاب قادرين على أن يصبحوا مشيًا للسياح العرب والطلاب قادرة على تصلى أن تصبح دبلوما وقادرة على أن تصبح كاتبة وباحثة.

التحديات التي تواجه معلمي اللغة العربية في القرن الحادي والعشرين في إندونيسيا هي: - هناك العديد من مدرسي اللغة العربية الذين لا تزال مهاراقم اللغوية ضعيفة شفهياً وخطياً. معلمو اللغة العربية ليسوا جزئياً من علماء اللغة العربية. معظم الأحكام التي حصل عليها مدرسو اللغة العربية في الكلية هي اللغة العربية النظرية

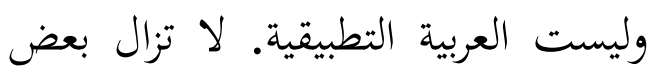
المدارس والمدارس الداخلية تركز على تعلم اللغة العربية، بدلاًا من تعلم كيفية استخدام اللغة العربية. من المرجح أن يقوم 
التفكير عالية المستوى والتقييم الأصيل للمعلمين إعداد إدارة تعليمية مفصلة. هذا يستنزف بالتأكيد طاقة المعلم والوقت. ركز المعلم أيضًا على الأهداف والأشياء التعليمية التي تؤدي إلى المجالات العاطفية والإدراكية والحركية. نظرًا للعدد الكبير من الحن إدارات التعلم التي يجب على المدرسين ترتيبها، يركز معلم اللغة العربية على إعداد الإدارة وإعداد التدريس، بالإضافة إلى لى إعداد المعلم لنموذج تقييم اللغة العربية. وهذا يعني أن المعلم منشغل بالتحضير بدلاً من عملية التعلم.

لتوضيح التحديات التي تواجه معلمي اللغة العربية في القرن الحادي والعشرين في إندونيسيا كما يلي:

\section{تطور اللفات العبية ومجال تعليمها} تطوير التكنولوجيا ، وهي العصر الصناعي 4.0 مطالبة في المناهج الدراسية

معيار كفايات معلمي اللغة العربية بإندونيسيا في القرن الحادي والعشرين في

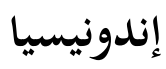

الإنترنت. يتعلم الطلاب اللغة العربية مع

مدرسهم على الإنترنت بما يكفي إما

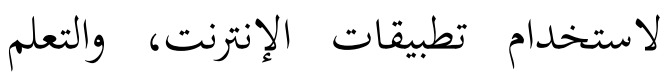
الإلكتروني، وفصول google، وميزات whatsapp الفصول الافتراضية، وبجموعات وما إلى ذلك. تعلم اللغة العربية مع اللغة الأم أسهل عبر الإنترنت، وتعلم اللغة العربية على يوتيوب والدورات عبر الإنترنت. على

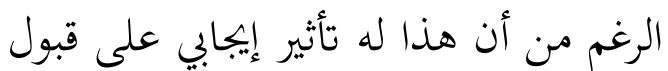

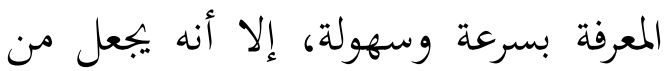

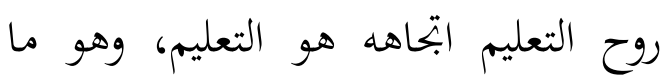
ينبغي القيام به مع تحول الوجه إلى العالم

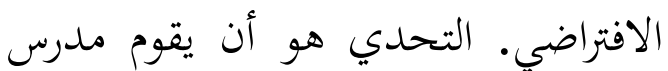
اللغة العربية بترقية مهاراته في التكنولوجيا. بالتأكيد يصبح هذا صعبًا إذا كان المعلم كبيرًا بالفعل، ومعظمهم كسول لتعلم التكنولوجيا. لا يستبعد إمكانية وجود مدرس لغة عربية شاب ما زال بحاجة إلى

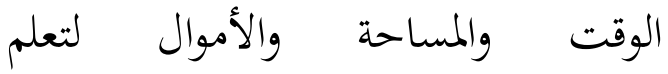

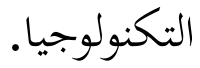
- مطالب في المناهج الدراسية لا تزال التغييرات في منهج ب ا • Y لها تأثير قوي على عنصر التعلم، وخاصة المعلمين. يتطلب تعقيد عملية التعلم العلمي التي تشير إلى تعلم مهارات 
الوصول إلى المواد من الإنترنت والبرامج التعليمية. يجب أن يكون المعلم أيضًا قادرًا على تقديم المواد ذات المشكلات الحالية. يجب أن يكون مدرسو اللغة العربية قادرين على التحدث باللغة العربية شفهياً في الفصول وخارج الفصول الدراسية. قادرة على لقلى نقل المواد باستخدام اللغة العربية المنطوقة والمكتوبة. يجب أن تتضمن جميع أنشطته أثناء التدريس قدرته اللفظية باللغة العربية. لمعرفة المعايير التي يجب أن تكون لدى كفاءات المعلمين بشكل عام، يمكن الاطلاع عليها في الرسم البياني التالي:

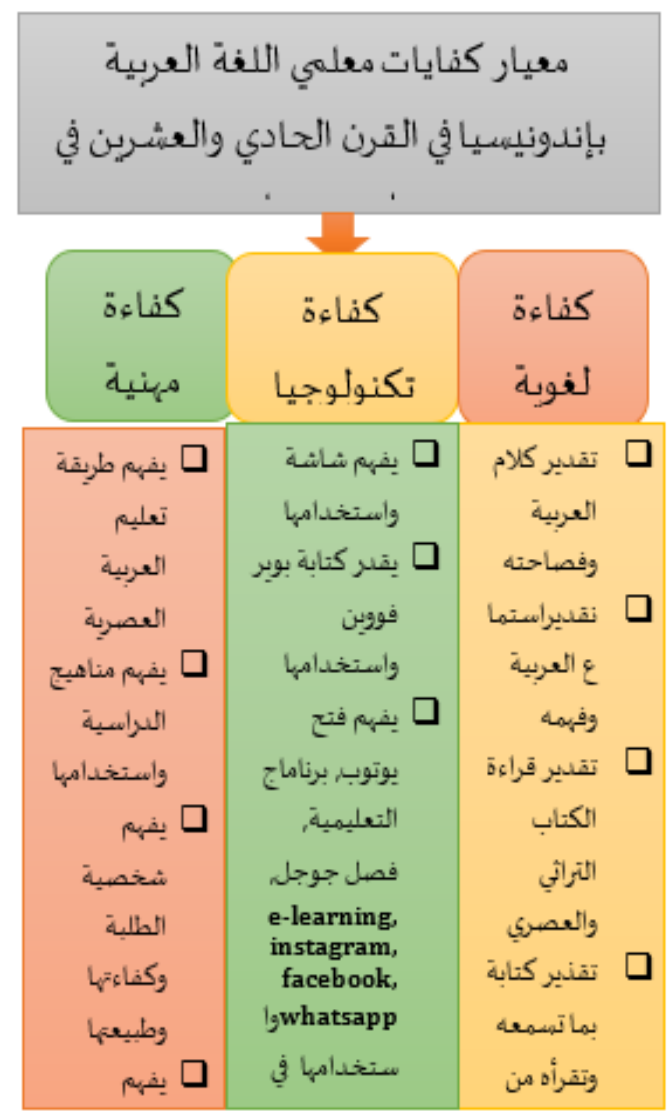

بشكل عام، يجب أن يكون

للمعلم أربع كفاءات، وهي الكفاءات المهنية

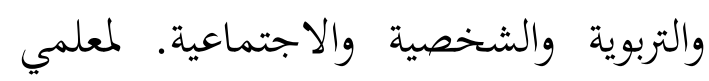
اللغة بالإضافة إلى الكفاءة اللغوية. لكن يجب الكباءية أن يكون المعلمون في القرن الحادي والعشرين

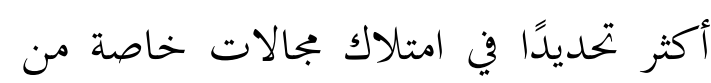

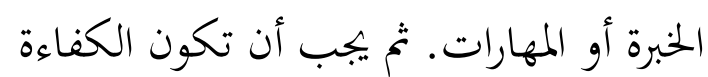

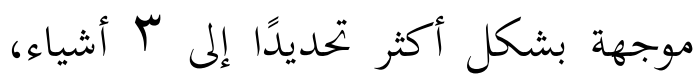

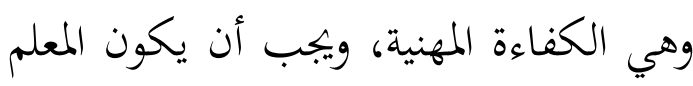
محترفًا في التدريس داخل الفصل وخارجه. يجب أن يتمتع المعلمون المهنيون بكفاءة المباة تعليمية جيدة وشخصية جيدة وحياة اجتماعية عالية. ثم يجب أن يكون لدى المعلم مهارات في مجال التكنولوجيا. يجب أن يكون المعلمون في القرن الحادي والعشرين قادرين على تشغيل أجهزة الكمبيوتر بشكل جيد، وأن يكونوا قادرين الكين على التدريس على الإنترنت باستخدام تطبيقات التعلم الافتراضية مثل الفصول الدراسية في Google وتطبيقات الوسائط

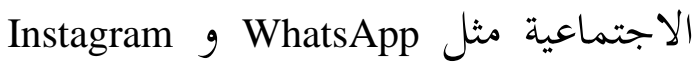
وما إلى ذلك. يجب أن يكون المعلم قادرًا على الى تقديم المواد بمساعدة powerpoint وشاشات الكريستال السائل بشكل مثير للاهتمام

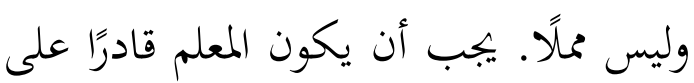


استراتيجية، لأن الشخص لديه المهمة الحقيقية للعملية الإنسانية والإنسانية والذكاء والحضارة وبناة الشخصية الوطنية. الاعتراف بهذه المهنة هو ولادة القانون رقم ع ا لعام 0 ه . . ب بشأن المعلمين والمحاضرين، كأساس قانوين للاعتراف

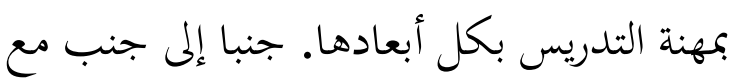
تطور العلوم والتكنولوجيا، دور المعلم آخذ في الاتساع، ليس فقط كما تسليم المواد والعلوم، ولكن أيضا جزء مهم من هذه العملية الإنسانية بكيث يتطلب المعلمون تغيير العقلية المرتبطة

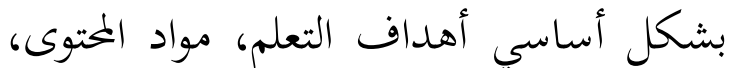
الأساليب ووسائل الإعلام، وتقييم التعلم. لم يقابل الاهتمام الكبير من الحكومة لمهنة التدريس بالكفاءة المعلم نفسه، وخاصة المعلم العربي الذي لا يزال منخفض الكفاءة. كفاءتا المنخفضة في الجوانب القدرة على نقل المواد، والجوانب قواعد النحو، النطق والكتابة. بالإضافة إلى ذلك هم ضعيف أيضًا في جوانب تقنيات التعلم واستخدام الوسائط. 7 كما أن اهتمام الحكومة تجاه مهنة التدريس أعلاه وكذلك المطالب متوازن مع الانتباه إلى رعاية المعلم. هناك أربعة مؤشرات

I'dadihi, wa Mutathallabat Tadribihi, Kairo: Alim al-Kutub, 2011, hlm. 20

Imtiyaz: Jurnal Pendidikan dan Bahasa Arab https://ejournal.iainbengkulu.ac.id/index.php/imtiyaz
الجهود التي يمكن بذلما في تطوير كفاءات

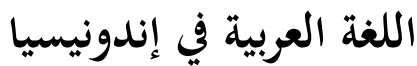

الاتجاهات العالمية التي يحتاج المعلمون

إلى معالجتها هي تعزيز الديمقراطية، حقوق الإنسان، الوعي البيئي، التعددية الدينية والثقافية، والسوق الحرة. على الرغم من أن الموضوعات عامة وعالمية، فإن تأثيراتا على ترثي تن الأنماط فكر المجتمع وسلوكه مهمان للغاية. للرد على هذا الاتحاه، عالم التعليم هو المكان الأول وقبل كل شيء. يبدو ابتحاهات التعليم في القرن الحادي والعشرين أكثر توجهاً نهو تطوير الإمكانات الموارد البشرية وهي الموارد البشرية كعاصمة للحضارة. هذا هو أساس الحكومة في

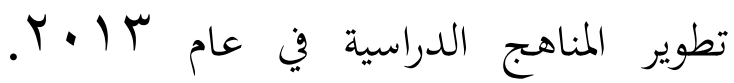
بالإضافة إلى التحديات المستقبلية معقدة بشكل متزايد للشعب الإندونيسي في عصر العولمة، وتحديداً منظمة التجارة العالمية وبجتمع الآسيان وأبيك و CAFTA ثم الكفاءات المستقبلية للرد على تحديات عصر العولمة هي ولي مهارات الاتصال. من بين الموارد البشرية التي عم الحكومة هي كفاءة المعلمين. لأن المعلم هي مهنة

${ }^{6}$ Kementerian Agama RI, Bahan Ajar PLPG Bahasa Arab tahun 2014, Jakarta, hlm. 11

7 Fadhlullah, Muhammad Rajab, Mu'allim al-Lughah al'Arabiyyah, Ma'ayir 
مستدامة حتى يتسنى للتعليم في إندونيسيا منافسة التعليم في بلدان في كل من آسيا وأوروبا. يمكن أن يتم تطوير كفاءات معلمي اللغة العربية من خلال المنظمات المهنية التي تم تشكيلها وتطويرها بواسطة مراقبي ومحبي اللغة العربية ، وهي اتحاد مدرسي اللغة العربية $\wedge$.IMLA

يتم تنفيذ تحسين كفاءة المعلمين من خلال استراتيجيات مختلفة في شكل المعلمين التعليم والتدريب (التدريب) وليس التدريب، من بين أمور أخرى على النحو التالي. ا ـ التعليم والتدريب، في شكل: أ. التدريب التحو النا. الداخلي التدريب الخارجي. IHT هو التدريب الذي يتم داخليًا في

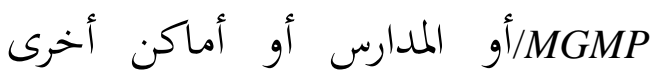
مصممة على تنظيم التدريب ، ب. برنامج التدريب. برنامج التدريب المهني هو تدريب يُجُرى في المؤسسات أو الصناعات ذات الصلة من أجل تحسين الكفاءة المهنية للمعلمين، ج. شراكة المدرسة. التدريب من خلال المدارس الشراكات يمكن أن يكون نفذت بالتعاون مع مؤسسات حكومية أو

Bahasa Arab dan Kebahasaaraban, Vol 1, No. (1), 2014, hlm. 5
استجابة الحكومة لرفاه المعلمين، وهي أولاً، إعلان المعلمين كمهنة بواسطة سوسيلو بامبانج يودويونو، الرئيس السادس لجمهورية إندونيسيا

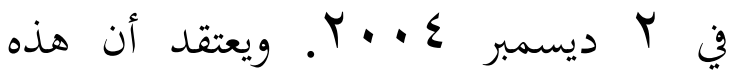
السياسة قادرة على تحسين رفاه المعلمين وكذلك مطالب المؤهلات وكفاءة المعلم. ثانياً، سن

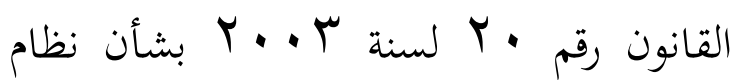
التعليم الوطنية. من خلال هذا القانون، يتم تنظيم حقوق والتزامات المعلمين في إطار الرعاية الاجتماعية كفاءة المعلم. ثالثًا، ولادة اللائحة الحكومية رقم 9 ( لعام 0 × . ب بشأن المعايير التربية الوطنية التي تتعلق أيضا بالكفاءة، وإصدار الشهادات، رفاه المعلم. والرابع، القانون

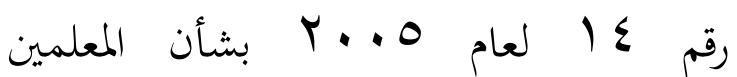
والمحاضرين الذين لديهم صدر في 7 ديسمبر 0. . . . يتناول هذا القانون أيضًا المؤهلات والشهادات ورفاه المعلم. من الوصف أعلاه، تصبح كفاءة المعلم قيمة مساومة لا يمكن إنكارها من أجل تحسين تقدم عالم التعليم. لذلك، تعد زيادة كفاءات المعلمين وتعزيزها أمرًا في غاية الأهمية حتى تكون هناك حاجة إلى طرق فعالة وضرورية

${ }^{8}$ Muhbib Abdul Wahab, Peran Bahasa Arab dalam Pengembangan Ilmu dan Peradaban Islam, Arabiyat : Jurnal Pendidikan 
الرسمية، وتناوب مهام التدريس، وتوفير مهام داخلية إضافية، والمناقشات مع الزملاء وما ولمات شابه ذلك، ح. التعليم الإضافي. تعزيز مهنة

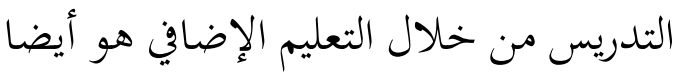
بديل لتعزيز مهنة التدريس في المستقبل. ץ. أنشطة أخرى غير التعليم والتدريب، وهي:

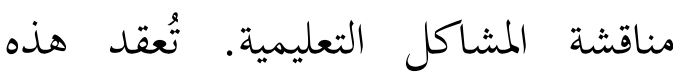
المناقشة بانتظام مع مواضيع وفقًا للمشاكل التي تواجه المدرسة، ب. الندوة. يمكن أن تكون مشاركة المعلمين في الندوات وتعزيز المنشورات العلمية أيضًا نموذجًا للتطوير المستمر لمهنة التدريس في زيادة كفاءة المعلم، ج. ورشة عمل. يتم تنفيذ هذا النشاط لإنتاج منتجات مفيدة للتعلم وتحسين الكفاءات والتطوير الوظيفي. يمكن إجراء ورش العمل على سبيل المثال في أنشطة إعداد SBC، وتحليل المناهج الدراسية، وتطوير المناهج الدراسية، وكتابة خطط الدروس، وما إلى ذلك، د. البحث، وكتابة الكتب أو المواد التعليمية، وصناعة وسائط التعلم، وعمل التكنولوجيا أو الأعمال الفنية. لتوضيح الأشياء أعلاه يمكن أن ينظر إليه في الرسم البياني التالي:
خاصة في بعض الخبرات، د. التعلم عن بعد. يمكن إجراء التدريب من خلال التعلم عن بعد دون تقديم المدربين والمتدربين في مكان واحد معين، ولكن مع نظام التدريب عبر الإنترنت وما شابه ذلك ، هـ. التدريب المتدرج والتدريب الخاص. يتم تنفيذ هذا النوع من التدريب في مركز التعليم والتدريب

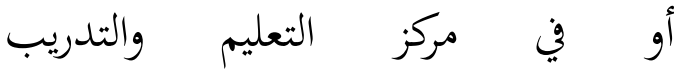
أو أتابع لوزارة الدين و P4TKdiklat)

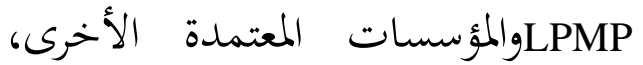
حيث يتم ترتيب برامج التدريب في مراحل تبدأ من المستويات الابتدائية والثانوية والمتقدمة والعالية. يتم ترتيب مستويات التدريب على أساس مستوى الصعوبة ونوع

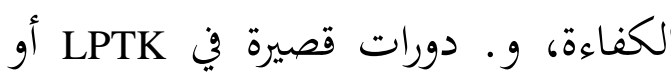
المؤسسات التعليمية الأخرى. تحف الدورات القصيرة في LPTK أو غيرها من المؤسسات التعليمية إلى تدريب تحسين كفاءة المعلمين في العديد من القدرات مثل إجراء البحوث العملية الصفية، وبتميع العمل العلمي، والتخطيط، وتنفيذ وتقييم التعلم ، وما إلى ذلك ، التدريب الداخلي للمدارس. يتم تنفيذ هذا التدريب الداخلي من قبل مدير المدرسة والمدرسين الذين لديهم السلطة لتعزيز، من خلال الاجتماعات 


\section{كفاءة اللغة, وتكنولوجيا, ومعرفي<smiles>[Al][V]</smiles> \\ Daftar Pustaka}

Abdul Wahab, Muhbib. 2014. ,Peran Bahasa Arab dalam Pengembangan Ilmu dan Peradaban Islam, Arabiyat : Jurnal Pendidikan Bahasa Arab dan Kebahasaaraban Vol 1 (1)

Al-Furqan, Muhammad Rizal. Musykilat Ta'lim al-Lugah al'Arabiyyah min Jiha Kafa'ah alMudarrisin ,Qillah Mudarrisy al-'Arabiyyah al-Akiffa' bi al-Maharat al-Lugawiyah', Artikel pada Majmu'ah Buhuts ,AlLugah al-'Arabiyyah Asas alTsaqafah al-Insaniyyahe, Jilid I, PINBA IMLA ke-9 27-29 Agustus 2015.

Eliya, Ixsir and Sodik, Achmad Ja'far (2019) PENGUATAN

KEILMUAN MAHASISWA MELALUI GERAKAN SADAR LITERASI DALAM UPAYA

MENANGKAL

RADIKALISME DAN BERITA HOAKS

DI MEDIA SOSIAL. In: International

Seminar on Islamic Studies, 28

Maret 2019, IAIN Bengkulu.

http://repository.iainbengkulu.ac.id

lid/eprint/2961
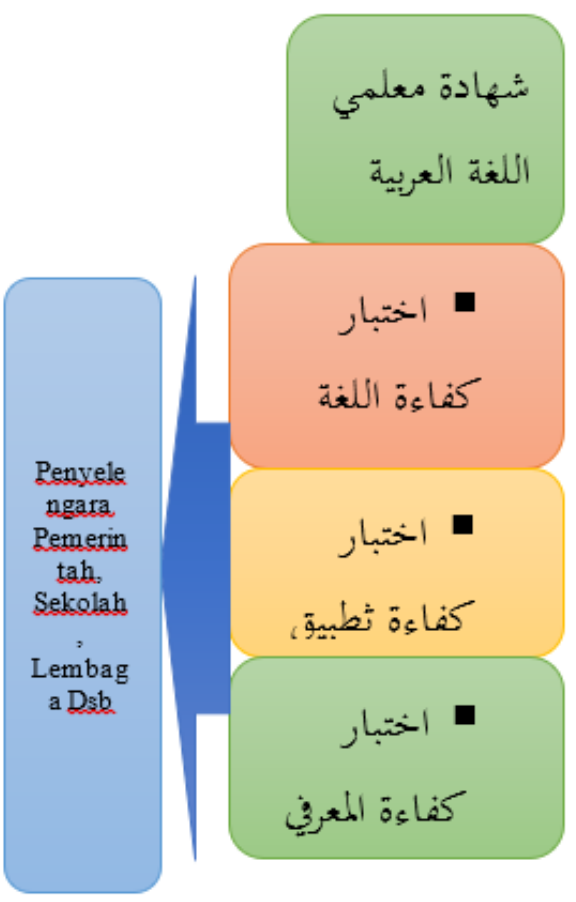

\section{الحاتمة}

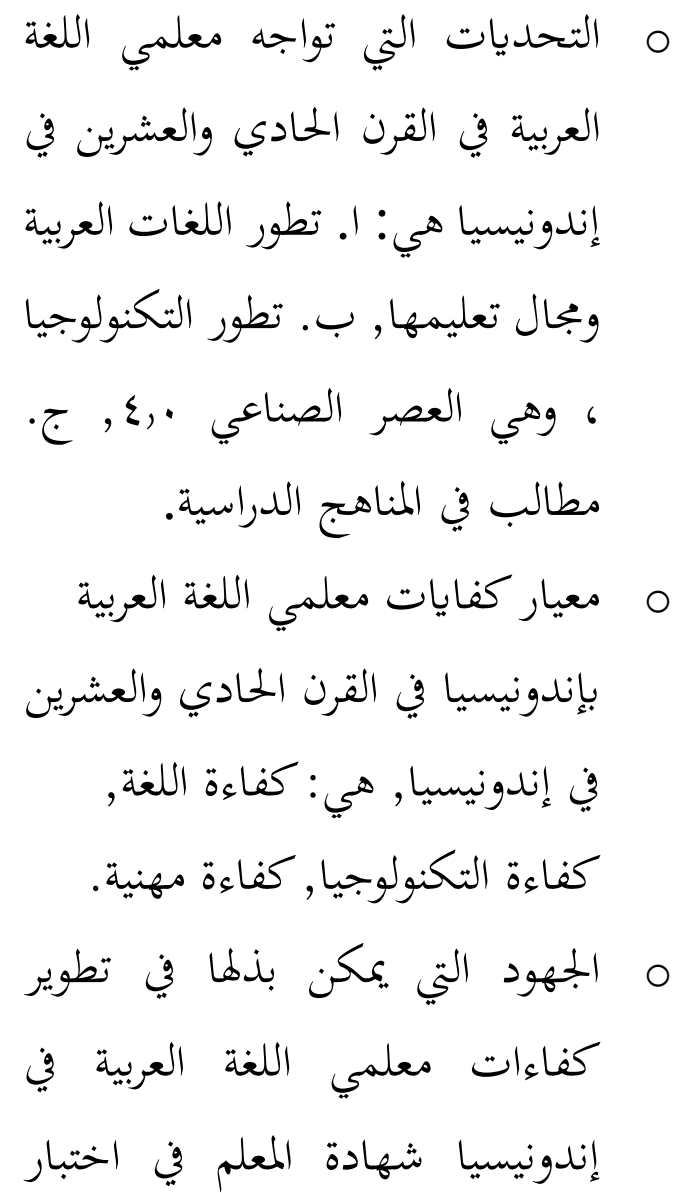


H. Hamzah. 2014. Profesi Kependidikan, Jakarta: Bumi Aksara.

Kementerian Agama RI, Bahan Ajar PLPG Bahasa Arab tahun 2014.

Kunandar. 2007. Guru Profesional, Jakarta: Rajawali Press.

Suyanto, Tantangan profesional guru di era global, Pidato Dies Natalis ke 43, UNY, 2007.

http://www.unismuh.ac.id/wp content/uploads/2018/05/Tantanga

n-Guru-Generasi-Z-dan-

Pembelajaran-Abad-21.pdf

https://www.silabus.web.id/pengertianempat-pilar-pendidikan/
Emzir, ,Kebijakan Pemerintah Tentang Pembelajaran Bahasa Arab di Madrasah dan Sekolah Umum', Makalah disampaikan dalam Pertemuan Ilmiah Nasional Bahasa Arab (PINBA) V di Bandung pada tanggal 23-25 Agustus 2007.

al-Fauzan, Abdurrahman. dkk. 1425 H. Durus al-Daurat al-Tadribiyah li Mua'allimi al-Lugah alArabiyah li Ghairi al-Natihiqin Biha (al-Janib al-Nazhari) , t.t.: Mu'assasah alWaqf alIslami.

Fadhlullah, Muhammad Rajab. 2011. Mu'allim al-Lughah al'Arabiyyah, Ma'ayir I'dadihi, wa Mutathallabat Tadribihi, Kairo: Alim al-Kutub. 